\title{
EDIFICIO PARA LA NUEVA SEDE SOCIAL DE LA EMPRESA NACIONAL DE ELECTRICIDAD, S. A. MADRID/ESPAÑA
}

\section{(BUILDING FOR THE HEADQUARTERS OF THE NATIONAL ELECTRICITY COMPANY)}

\author{
Eleuterio Población, Dr. Arquitecto \\ Adaptación del texto: José Maria Jenaro Garrido, Arquitecto
}

\section{RESUMEN}

En el presente articulo se describe detalladamente el proyecto y ejecución del edificio ENDESA, en Madrid, compuesto por dos edificios de doce alturas uno, y dos el otro, unido por un espacio ajardinado.

En céntrica situación ha exigido una especial adaptación a las Ordenanzas Municipales en cuanto a condiciones de volumen y uso.

Entre sus caracteristicas más notables pueden señalarse la sujeción a una red modular, la adecuación del espacio a las actividades que en él se desarrollarán y las soluciones adoptadas para su cerramiento.

\section{SUMMARY}

In this work, the design and execution of ENDESA building, in Madrid, is fully described. It is a compound of a

twelve-storeyed and a two-storeyed buildings, separated by a garden.

Being placed in a central district, a special adaptation to the bylaws was necessary concerning the volume and use conditions.

Among its most remarkable characteristics it could be pointed out the subjection to a modular frame, the adequacy of the spaces for the activities that are to be developped in it and the adopted solutions for its closure.

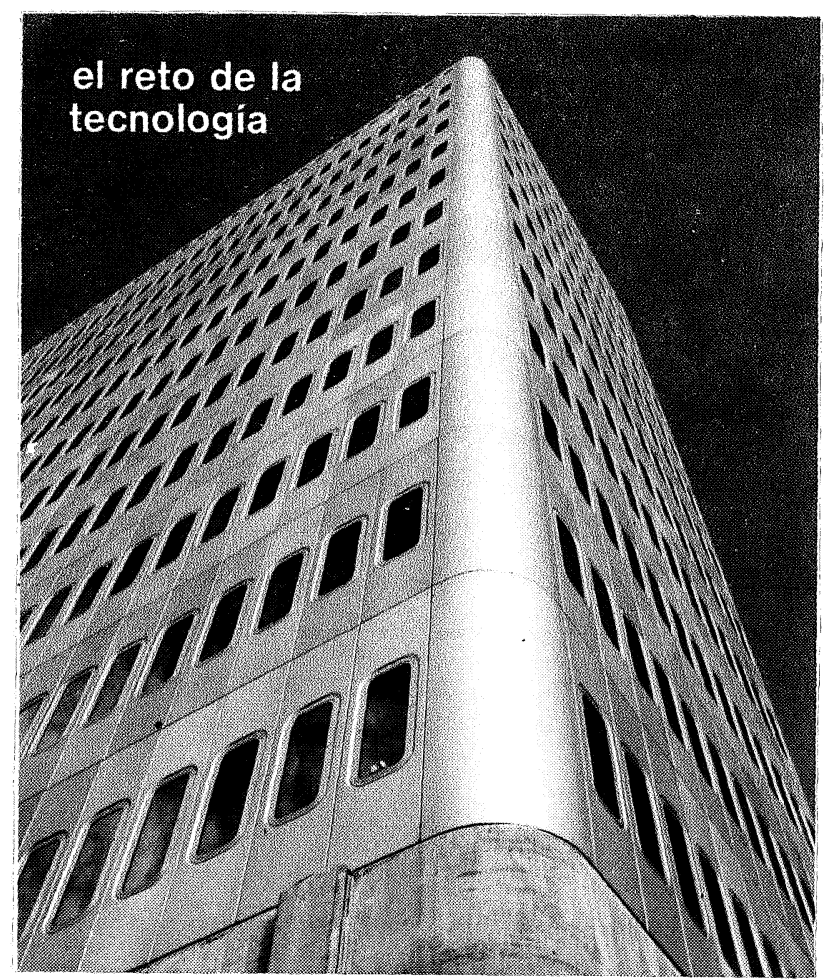

\section{TIPO DE PROYECTO}

Se trata de un conjunto de nueva planta compuesto por dos edificios, de doce alturas uno, y de dos el otro, unidos por un espacio ajardinado, y destinados a uso de oficinas y necesidades anejas con aparcamiento subterráneo.

\section{EMPLAZAMIENTO DEL SOLAR Y ORDENANZAS QUE LE AFECTAN}

El solar de este edificio queda emplazado en la Parcela n.o 53 del Proyecto de Ordenación de la Prolongación de Príncipe de Vergara y se sitúa en la acera de los números impares de una importante via madrileña de reciente apertura.

La parcela queda afectada por la Ordenación de la Prolongación de Principe de Vergara en cuanto se refiere a condiciones de volumen -altura, patios, entrantes, salientes y vuelos, etc.-y uso.

La ordenación de volúmenes prevista en el Proyecto Municipal es de manzanas integradas por edificación abierta, rodeada de espacios libres. 


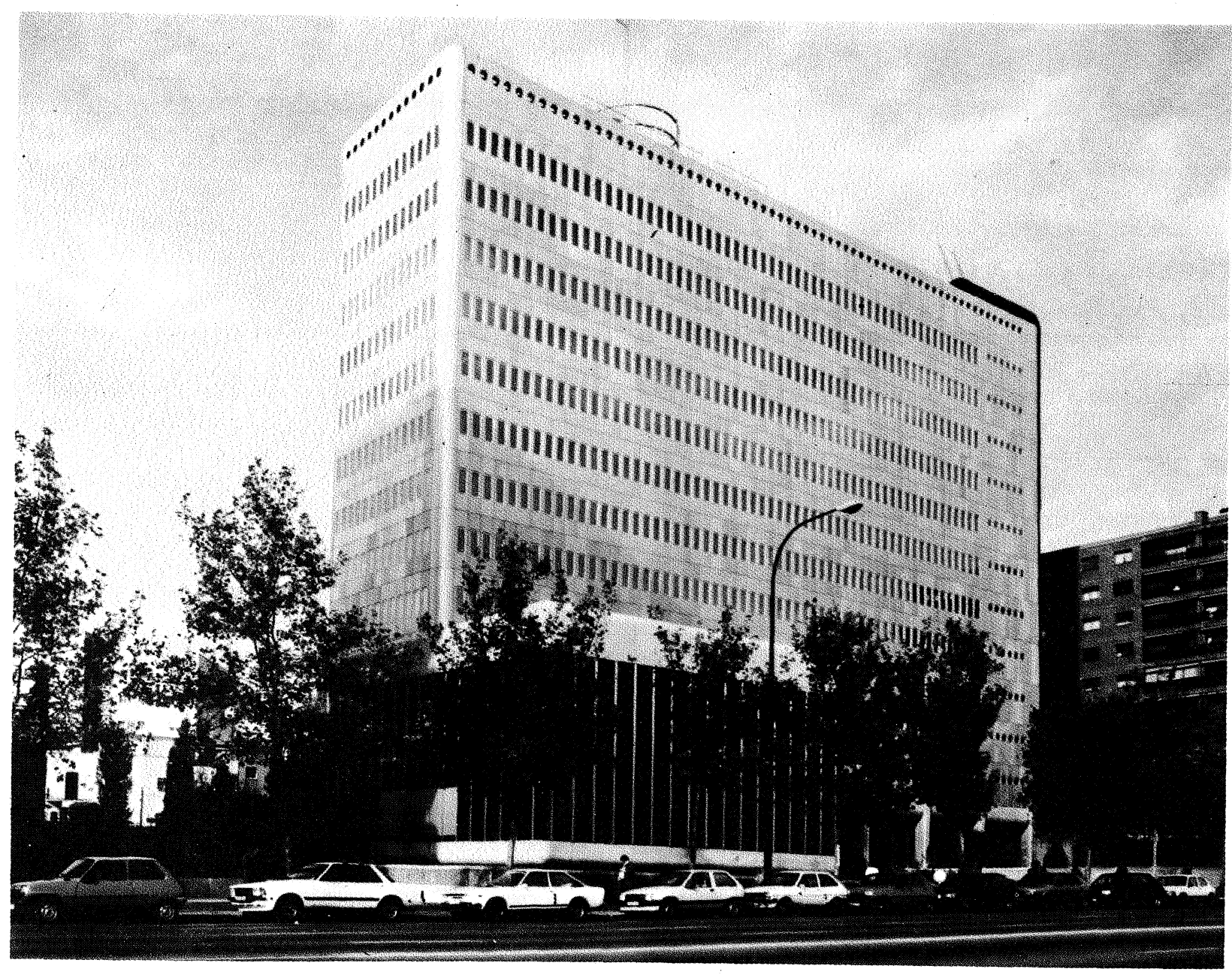

Para el resto, el solar queda afectado por las Ordenanzas Generales Municipales vigentes.

\section{PREMISAS DE PLANTEAMIENTO $Y$ PROGRAMA DE NECESIDADES}

El programa a desarrollar, facilitado por la entidad propietaria y discutido a lo largo de los distintos esquemas de planteamiento y croquis, es el que se transcribe a continuación:

a) Utilización máxima de las plantas para las necesidades de oficinas, con la máxima elasticidad de distribución.

b) Zonificación de la planta tipo en dos áreas: una para la dirección y atención exterior y otra de trabajo de equipos.

c) Reducción al minimo de los servicios sanitarios y de otro tipo, de estas plantas. Los cuales se complementarán con los establecidos en la zona de cafeteria.

d) Previsión de los espacios y de las condiciones necesarias para el establecimiento de los siguientes servicios:
- Sala de telefonia y radio...... $100 \mathrm{~m}^{2}$

- Sala de Ordenadores ........ 160 m² $^{2}$

- Sala de Control de la Red..... . 150 m² $^{2}$

- Sala de alimentación tensión segura ................ $60 \mathrm{~m}^{2}$

e) Cafeteria para desayunos y almuerzos del personal, capaz para servir al total de los ocupantes del edificio en tres turnos.

f) Sala de conferencias y reuniones para unas doscientas cincuenta personas.

g) Aparcamiento en el mayor número posible.

h) Archivo General.

\section{JUSTIFICACION DE LA SOLUCION ADOPTADA}

Partiendo de la necesidad de conseguir la necesaria elasticidad de distribución y programación ajustándose a una trama modular reguladora, se planteó el dificil asunto de la elección del módulo. 


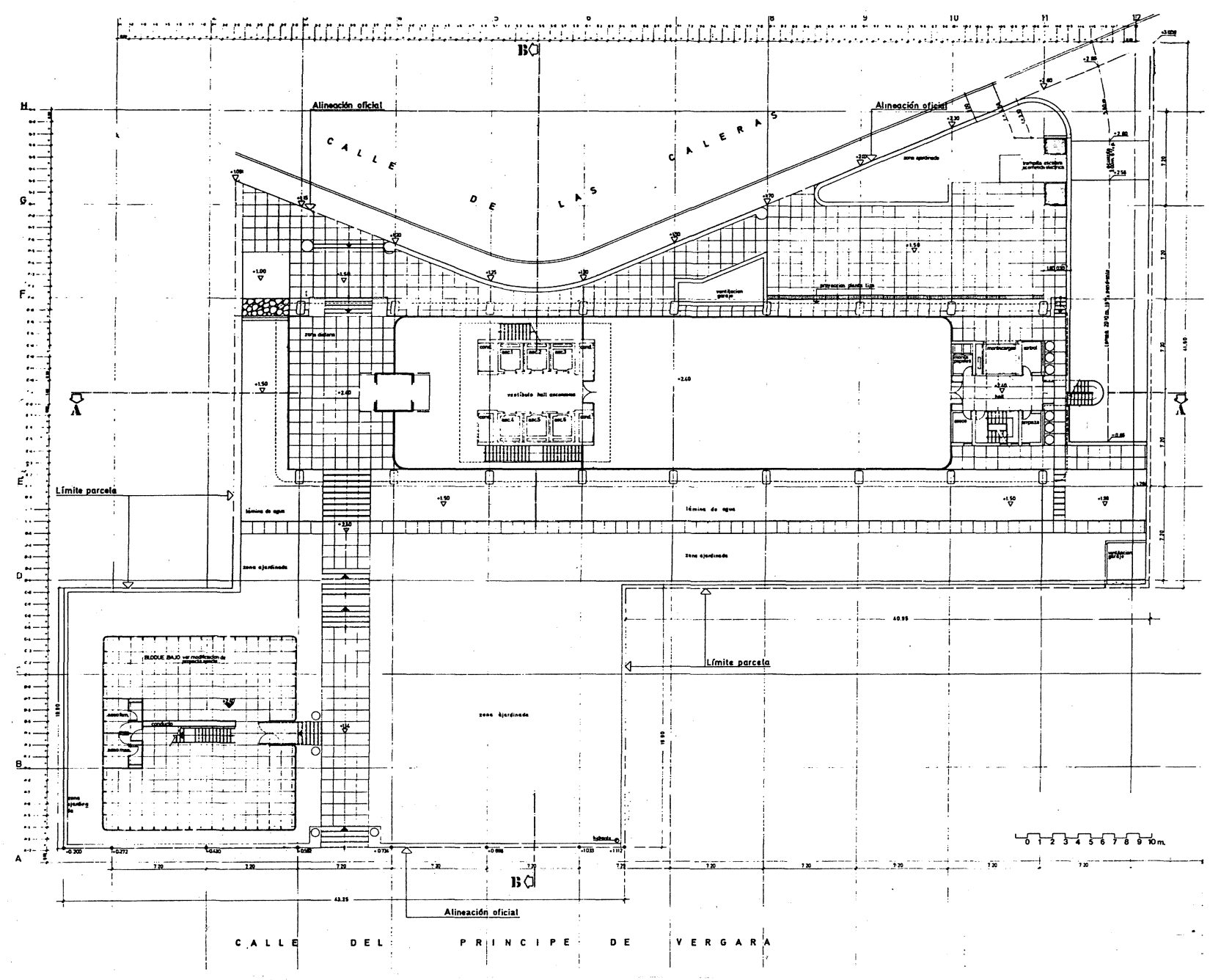

La experiencia y la poca prefabricación existente en la zona han conducido a una única alternativa en este dilema: el módulo base de $90 \mathrm{~cm}$ y el de $120 \mathrm{~cm}$, ambos múltiplos del pie decimal de 30 $\mathrm{cm}$, útil para establecer una relación entre la medida del cuerpo humano con nuestro sistema métrico y por ende con la construcción de que se trata.

Los dos módulos son igualmente válidos en lo que se refiere únicamente a la planta, pero el de $90 \mathrm{~cm}$, por ser menor, permite mayor número de combinaciones, y si se extiende la trama modular en las tres direcciones del espacio, el más apto, dentro de las medidas que las Ordenanzas imponen resulta ser el de tres pies, o $90 \mathrm{~cm}$ ya que origina las alturas idóneas para los distintos usos, es decir $3 \mathrm{M}=2,70$ metros para oficinas, 5 $M=4,50$ metros para zonas nobles de recepción, $1 \mathrm{M}=0,90$ metros para gruesos entre los pisos -forjado e instalaciones- 2,5 M $=2,25$ metros para pasos y servicios, etc.

Asi, éste ha sido el módulo elegido y por tanto el que domina y concreta todo el contexto del Proyecto.
Dado que las alineaciones quedan fijadas en el Plan Parcial, el establecimiento de la trama reguladora se ha conseguido mediante la adaptación a ella del edificio más importante por volumen, lo cual se ha conseguido a base de actuar con los entrantes, salientes y vuelos autorizados y mediatizando la implantación del resto a esta imposición primaria.

Esto obligó a desplazar levemente el edificio de dos plantas de forma que adaptándose a la trama reguladora no produjese anomalia a lo previsto en el Plan, ni, por supuesto, perjuicios a terceros.

\section{ANALISIS MORFOLOGICO}

Lamentablemente la necesidad de adaptación de las posibles soluciones a las exigencias de los Planos Especiales que se redactan, en las que quedán prácticamente determinados los volúmenes en su aspecto formal primario, no siempre permite al arquitecto elegir la solución idónea y limita.su especulación a hallar la que cree más conveniente dentro de una forma previa que le mediatiza. 


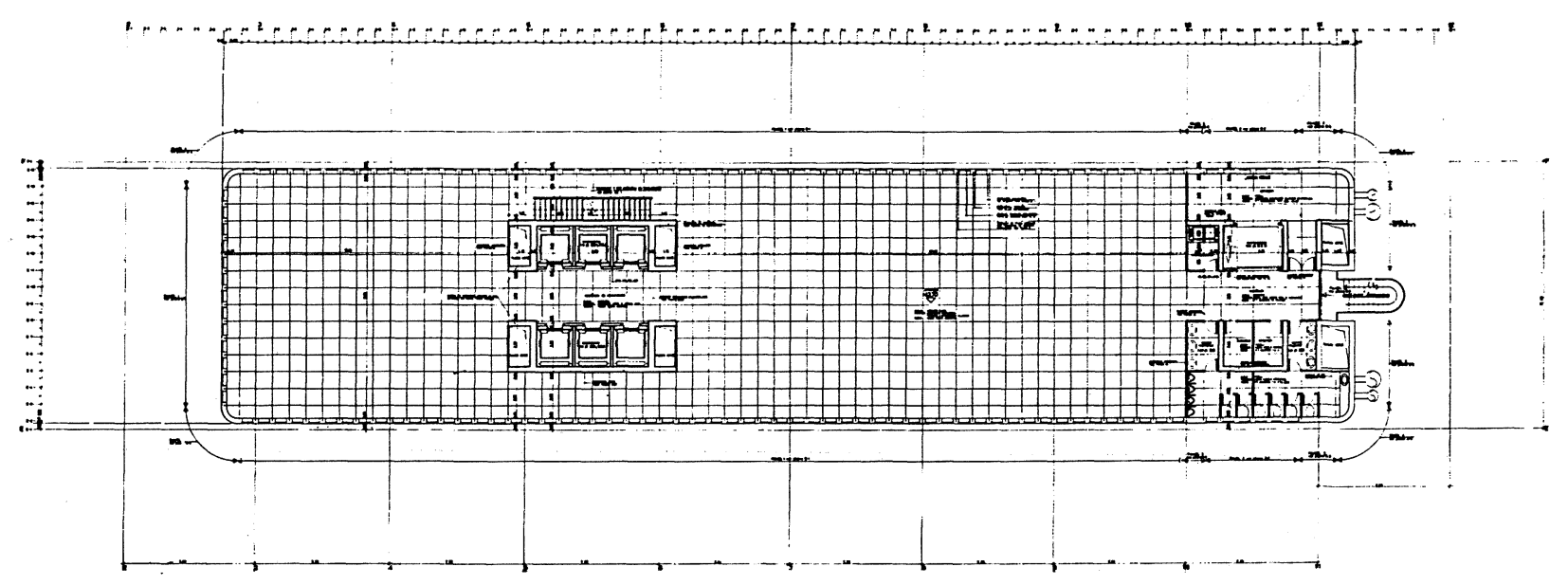

Planta tipo

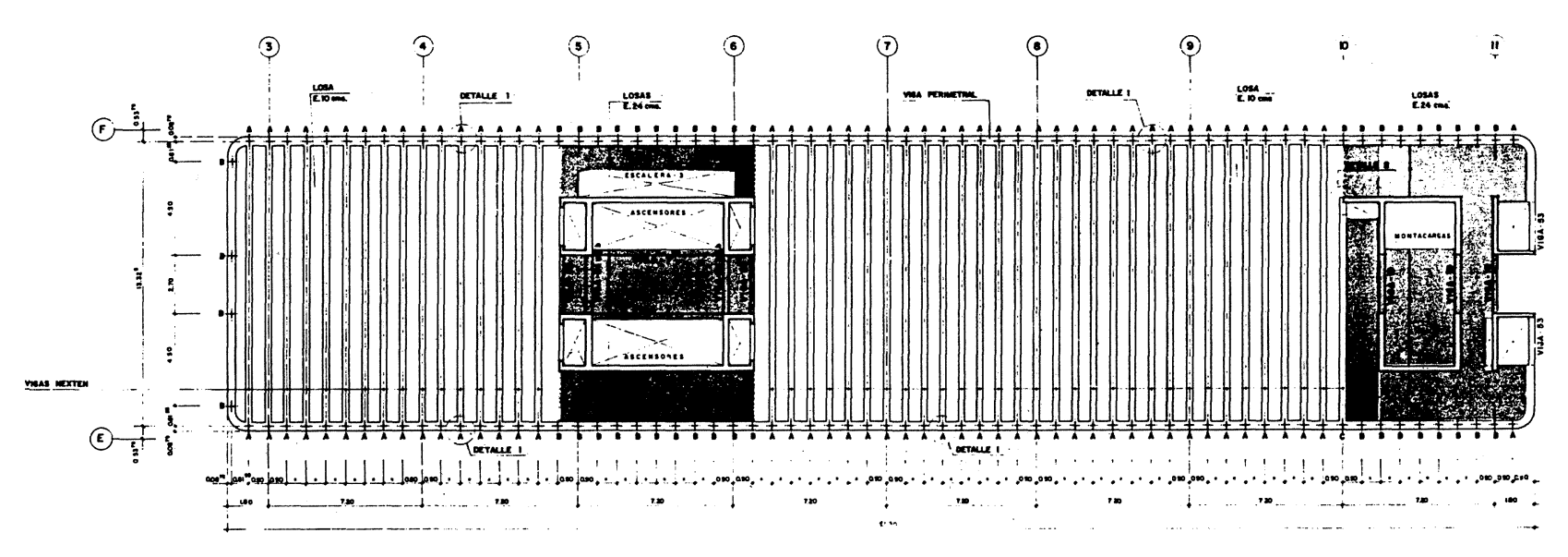

Planta de estructura de 2.a a 8 .a

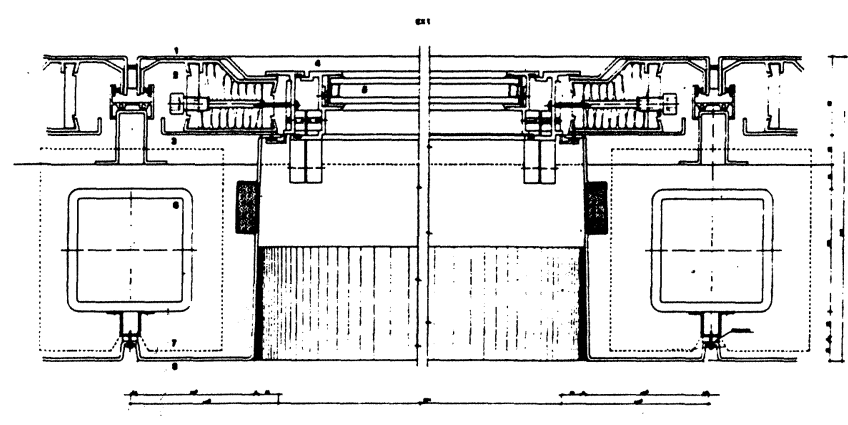

Detalle de fachada

1. Aluminio estampado $3 \mathrm{~mm}$. - 2. Perfil con ruptura puente termico. 3. Acero galvanizado. - 4. Carpinteria de aluminio extrusionado. - 5. Acristalamiento. - 6. Pilar metalico. - 7. Ignifugado con vermiculita. 8. Panel de moldeado plastico.
En nuestro caso, la adaptación de los condicionantes modulares a la planta prevista no han sido difíciles pero han obligado a sacrificar 0,30 metros en la anchura total que se compensan con los vuelos laterales en el edificio principal.

La voluntad de expresionismo funcional -en que se incluye la necesidad de representatividad del conjunto- unido a la resolución del problema que plantea la posición en el solar del volumen principal, situado lejos del acceso más importante, han llevado a levantar este elemento, sobre un basamento transparente, tanto como lo han permitido las normas acentuando el efecto de acercamiento mediante la creación de un jardin en pendiente que acorte la perspectiva. 
El edificio se concibe como un contenedor de una actividad que debe desarrollarse en las mejores condiciones que las actuales técnicas permitan. $Y$ ese uso de la técnica se quiere que trascienda al exterior. El cerramiento de este gigantesco contenedor de actividades debe conseguir aislarla del exterior tanto como lo permitan, no las condicionantes de iluminación directa -que no se consideren fundamentales en este caso-, sino las sicológicas provinientes de la necesidad de no sentirse ausente del entorno físico del edificio.

Entre los cerramientos ligeros -únicos válidos para el planteamiento general previsto- el muro cortina procede de una técnica primaria enraiza- da con formas de la arquitectura popular. No corresponde al nivel actual de desarrollo tecnológico que se observa a nuestro alrededor en nuestros vehiculos: automóviles, aviones, trenes.

Se ha elegido, pues, la misma técnica que utilizan los medios de transporte: la estampación o embutición. En cada panel se produce el hueco y el macizo necesarios para cumplir su función -visión, aislamiento- en las óptimas condiciones de ensamblaje al conjunto.

El cerramiento de plantas de oficina se ha diseñado entonces a base de paneles prefabricados de aluminio anodizado de $0,90 \times 3,50 \mathrm{~m}$, en los que se incluye la carpinteria del mismo material formando ventanas pivotantes de eje horizontal

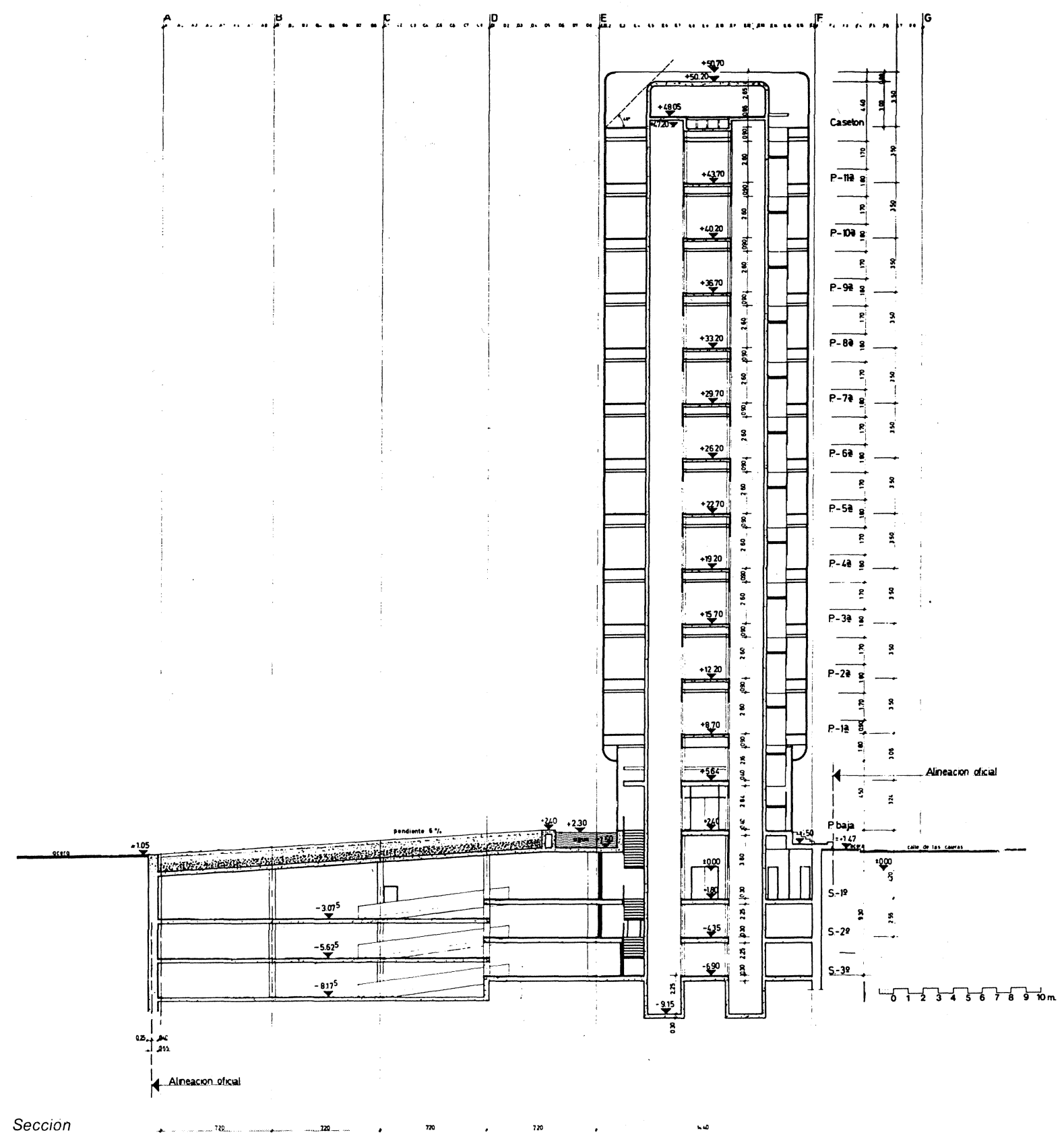




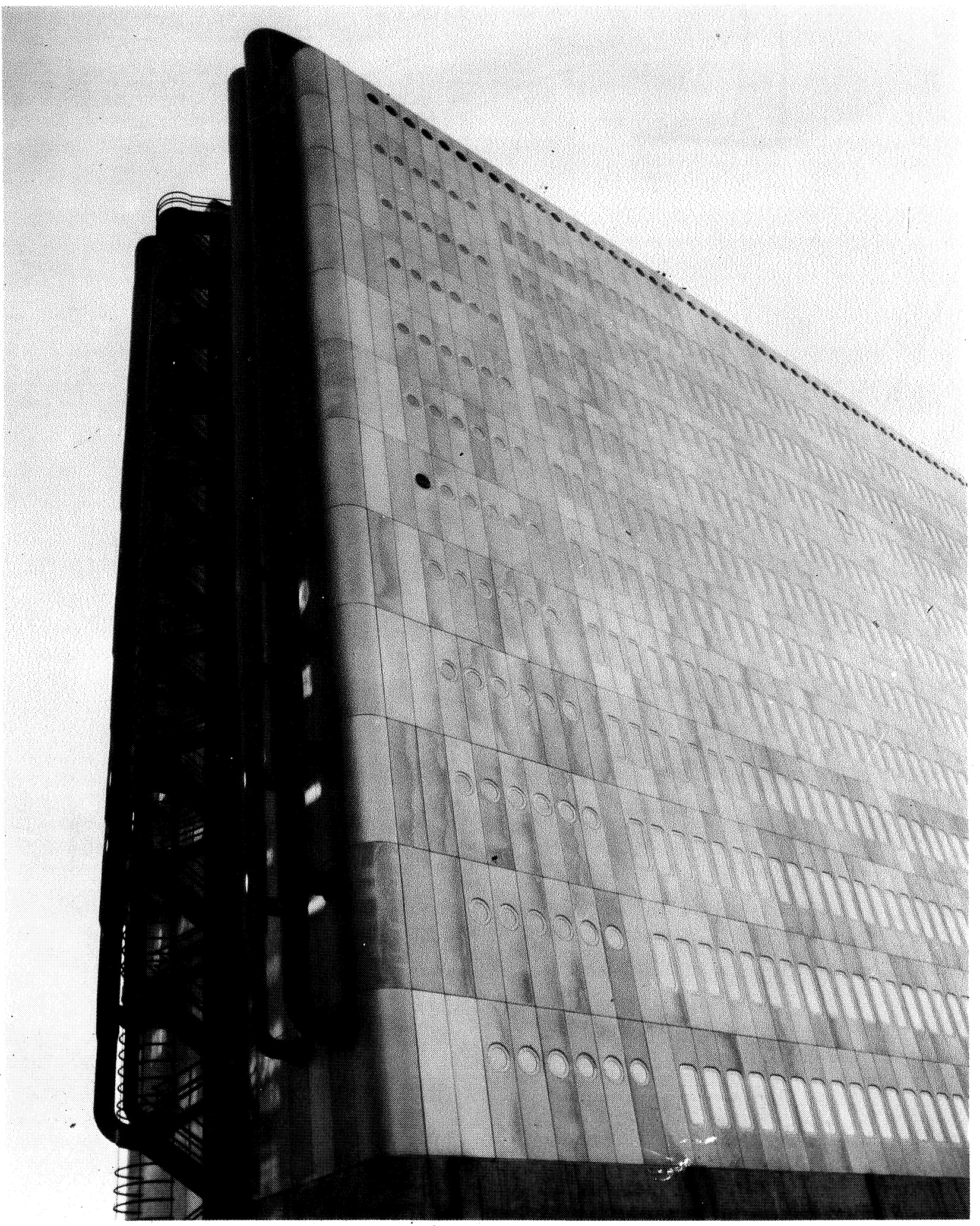


de $0,60 \times 1,50 \mathrm{~m}$. Asimismo incluyen un aislamiento térmico de $10 \mathrm{~cm}$ de Poliuretano expandido por inyección.

El interior ha sido previsto con paneles moldeados en plástico de $0,90 \times 2,60 \mathrm{~m}$.

Todo el conjunto tiene un espesor de $30 \mathrm{~cm}$ en los que quedan embebidos los pilares metálicos de fachadas con sus respectivas protecciones contra incendios.

Esta técnica permite ser utilizada también en el edificio secundario, puesto que no tiene prácticamente limitación formal.

Las caracteristicas, garantias, tolerancias y protecciones que debe cumplir el aluminio de los paneles han sido establecidas en la memoria de proyecto.

Por este camino se ha llegado, pues, a poder coordinar un conjunto arquitectónico dificil por la disparidad dimensional de sus componentes.

Las formas curvilineas introvierten el prisma de oficinas y extrovierten el edificio de reuniones públicas.

Los acentos que proporcionan la apariencia exterior de estructura e instalaciones refuerzan el expresionismo técnico que se ha buscado.

\section{ADAPTACION A LAS ORDENANZAS}

Siendo este edificio, como se ha explicado antes, una construcción sujeta a las determinaciones estrictas de una trama modular en las tres direcciones del espacio, la aplicación de las alturas especificadas por las ordenanzas, normalmente válidas para otro criterio de composición, resulta dificilmente coordinable en este caso.

En efecto, debido al espesor anormal que alcanza la placa estructural de forjado por las grandes luces que salva, y a la presencia de las conducciones de las instalaciones, el espesor total de entrepisos resulta próximo a los $100 \mathrm{~cm}$ y así, aun cuando la altura libre utilizable es de 2,50 metros, la total es de 3,50 metros. Esta licencia en la interpretación puede estimarse que no afecta a la intención del urbanista.

En cuanto a la superficie total en el bloque principal, se ha compensado un pequeño exceso de cada planta con los espacios abiertos de la planta baja, de forma que el total construido en la solución adoptada es prácticamente el mismo que el previsto en la ordenación.

La estructura exterior está dentro de los gálibos previstos en las especificaciones de la ordenan$z a, y a$ que no sobrepasan los vuelos autorizados de 1,50 metros.
El tema de las rasantes también ha sido muv ponderado en el estudio que se presenta, dado que el solar de que se dispone ofrece una gran dificultad en este tema especifico al dar a dos calles con una diferencia de cota que alcanza los tres metros. Por esta razón el conjunto del sótano queda, en general, por debajo de la rasante de la calle de las Caleras y algo por encima de la rasante que corresponde a la calle Principe de Vergara. Se ha procurado en todo momento que esta altura del jardín sobre la rasante de la calle principal, no sobrepase la altura de la visual peatonal de tal forma que no quede desvirtuado el concepto de la ordenación que entiende este conjunto como una serie de bloques aislados de zona verde privada.

El bloque de dos plantas previsto en la ordenación con fachada a Principe de Vergara se ha acoplado a las coordenadas fijadas por la trama reticular estructural del bloque principal, por lo que no queda exactamente en la posición que se fija en la ordenación, pero sí con una desviación despreciable y muy aproximada a la prevista.

Se ha tenido en cuenta, tomándolas con exceso, cuantas normas son de aplicación para la prevención de incendios, tanto en lo que se refiere a ascensores, previstos con maniobra especifica para desalojo en caso de alarma y uso de una unidad para los bomberos, como en lo que se refiere a escaleras, cuyo dimensionamiento se ha hecho con generosidad.

Se han proyectado los espacios necesarios para la ubicación de una columna seca y otra húmeda por escalera, asi como la conveniente instalación de alarma por detección de humos en las plantas de oficinas, y por aumento de temperatura en los aparcamientos y distintas salas de máquinas.

Los aparcamientos tienen la ventilación natural que exige la actual normativa y una ventilación forzada de marcha automática en función del porcentaje de CO de la atmósfera de los garajes. Extintores y bocas de incendio se sitúan en el aparcamiento cubriendo las áreas reglamentarias.

\section{CONSIDERACIONES FUNCIONALES}

Entre las diversas funciones que se han de realizar en el conjunto se ha establecido una division de acuerdo con el siguiente esquema general:

1. Agrupar en el bloque de doce plantas toda la temática de producción laboral, relación con visitas, dirección y conjunto de administración, etc. ocupando, incluso, las zonas de sotano definidas por la estructura principal.

2. Utilizar el edificio de dos plantas para las funciones de restauración, descanso y representación. 
3. Potenciar el jardin, como nexo entre ambos edificios, asi como la primera planta de sótano, que ha de ser, en cierta manera, representativa.

Siguiendo este criterio general la planta del bloque de altura se ha zonificado claramente, mediante la adecuada disposición de los núcleos verticales, en dos áreas conexas pero independientes. La correspondiente al extremo Sur como zona de representación y dirección y la más amplia, que ocupa la parte central, a área de trabajo. De acuerdo con la coordinación dimensional impuesta por el módulo utilizado, cada una de estas áreas puede ser dividida en despachos, o bien, utilizarse como oficina paisajista o abierta.

El extremo Norte del bloque está ocupado por los servicios de planta -aseos y zona de café y refrescos- y por las áreas necesarias para el gran montacargas y los montapapeles.

La planta baja se ha procurado mantenerla lo más diáfana posible de forma que exprese al exterior la estructura funcional del bloque. Los extremos abiertos y la zona central acristalada en su totalidad-dedicada a exposiciones, maquetas, etc. de la Sociedad- conseguirán reflejar esta intención.

El bloque de dos plantas, en la entrada, contiene tres zonas diferenciadas, la planta baja, también muy diáfana y con clara intención formal, no es más que acceso; arriba, a través de dos escale- ras helicoidales, se accede a la sala de conferencias y actos públicos; hacia abajo se llega a la cafeteria restaurante establecida en el semisótano.

El acceso desde Principe de Vergara se ha enfatizado, como antes hemos dicho. La gran escalinata que se prolonga en el paso hacia el bloque principal siguiendo la pendiente del jardin, permiten la percepción del conjunto desde el principio. El estanque que rodea el edificio aligera la zona basamental y, por reflejo, alarga la imagen.

\section{ENCARGO DEL PROYECTO Y EQUIPO DE TRABAJO}

El proyecto de este edificio, que se redactó por encargo de ENDESA (Empresa Nacional de Electricidad, S. A.) fue concebido y dirigido en su extensión por el Dr. Arquitecto D. Eleuterio Población Knappe.

En sus distintas fases de realización han colaborado los arquitectos D. Luis Cuenca, D. Francisco Gregori y D. Luis de la Puente, asi como el equipo dirigido por E.P.K. para dicho trabajo.

Para los estudios correspondientes a Mecánica del Suelo y Estructuras se contó con la colaboración de O.T.E.P., S. A. y para los que se refieren a las distintas y complejas instalaciones del edificio intervino el equipo de A.V.C. Ingenieros Consultores, expertos en estas disciplinas.

\title{
publicación del i.e.t.c.c.
}

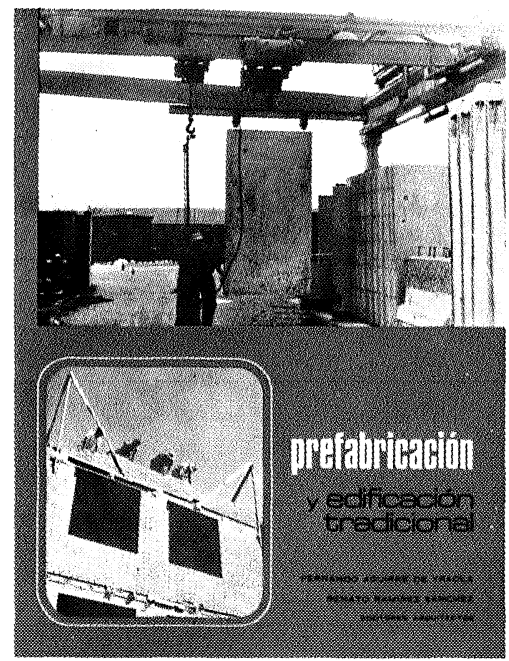

\author{
Fernando Aguirre de Yraola y Renato Ramírez Sánchez \\ Drs. Arquitectos
}

Industrialización de la construcción, en sentido general dentro de ésta, es el empleo, en forma racional y mecanizada, de materiales, medios de transporte y técnicas constructivas para conseguir una mayor productividad.

El proceso del desarrollo de la industrialización, que nos transformará el sector tradicional, o convencional, en otros más de acuerdo con nuestras exigencias actuales, deberá aplicarse no sólo al producto de este desarrollo, es decir, la edificación, sino también al conjunto de la producción.

El carácter acusadamente gráfico y esquemático de esta publicación pretende constituir una síntesis de parámetros básicos para la Racionalización, Normalización y Tipificación de elementos prefabricados, organización en obra y factoria, para el estudio económico entre construcciones convencionales e industrializadas.

Un volumen encuadernado en cartoné plastificado, de $21 \times 17 \mathrm{~cm}$, compuesto de 125 páginas, 25 cuadros y 56 flguras de linea. 